\title{
A FUNDAMENTAL MATRIX EQUATION FOR FINITE SETS ${ }^{1}$
}

\author{
H. J. RYSER
}

\begin{abstract}
Let $S=\left\{x_{1}, x_{2}, \cdots, x_{n}\right\}$ be an $n$-set and let $S_{1}$, $S_{2}, \cdots, S_{m}$ be subsets of $S$. Let $A$ of size $m$ by $n$ be the incidence matrix for these subsets of $S$. We now regard $x_{1}, x_{2}, \cdots, x_{n}$ as independent indeterminates and define $X=\operatorname{diag}\left[x_{1}, x_{2}, \cdots, x_{n}\right]$. We then form the matrix product $A X A^{T}=Y$, where $A^{T}$ denotes the transpose of the matrix $A$. The symmetric matrix $Y$ has in its $(i, j)$ position the sum of the indeterminates in $S_{i} \cap S_{j}$ and consequently $Y$ gives us a complete description of the intersection patterns $S_{i} \cap S_{j}$. The specialization $x_{1}=x_{2}=\cdots=x_{n}=1$ of this basic matrix equation has been used extensively in the study of block designs. We give some other interesting applications of the matrix equation that involve subsets with various restricted intersection patterns.
\end{abstract}

1. The matrix equation. Let $S=\left\{x_{1}, x_{2}, \cdots, x_{n}\right\}$ be an $n$-set (a set of $n$ elements) and let $S_{1}, S_{2}, \cdots, S_{m}$ be subsets of $S$. We set $a_{i j}=1$ if $x_{j}$ is a member of $S_{i}$ and we set $a_{i j}=0$ if $x_{j}$ is not a member of $S_{i}$. The resulting $(0,1)$-matrix

$$
A=\left[a_{i j}\right]
$$

of size $m$ by $n$ is the familiar incidence matrix for the subsets $S_{1}, S_{2}, \cdots, S_{m}$ of $S$. It is clear that $A$ characterizes the configuration of subsets.

Now let us regard $x_{1}, x_{2}, \cdots, x_{n}$ as independent indeterminates over the field of rational numbers and define

$$
X=\operatorname{diag}\left[x_{1}, x_{2}, \cdots, x_{n}\right] .
$$

We then form the matrix product

$$
A X A^{T}=Y \text {. }
$$

The matrix $A^{T}$ denotes the transpose of the matrix $A$. The matrix $Y$ is a symmetric matrix of order $m$. We know the structure of this matrix

Received by the editors August 25, 1971.

AMS 1970 subject classifications. Primary 05B20, 05B30; Secondary 15A24.

Key words and phrases. Block design, incidence matrix, indeterminate, matrix equation, set, set intersection, subset, $(0,1)$-matrix.

1 This research was supported in part by the Army Research Office-Durham under Grant DA-ARO-D-31-124-G1138 and the Office of Naval Research under Contract N00014-67-A-0094-0010.

(c) American Mathematical Society 1972 
explicitly. Thus the matrix $Y$ has in its $(i, i)$ position the sum of the indeterminates in $S_{i}$. More generally, the matrix $Y$ has in its $(i, j)$ position the sum of the indeterminates in $S_{i} \cap S_{j}$. It follows that the matrix $Y$ gives us a complete description of the intersection patterns $S_{i} \cap S_{j}$ for the subsets $S_{1}, S_{2}, \cdots, S_{m}$ of $S$. For related material concerning intersection patterns we mention here the earlier investigations of Goodman [1], Hall [2], Kelly [3], and Ryser [6].

The matrix $Y$ involves the indeterminates $x_{1}, x_{2}, \cdots, x_{n}$ and we write

$$
Y=Y\left(x_{1}, x_{2}, \cdots, x_{n}\right) .
$$

We may set $x_{1}=x_{2}=\cdots=x_{n}=1$ and then (1.3) reduces to the classical equation

$$
A A^{T}=Y(1,1, \cdots, 1) .
$$

But notice that $Y(1,1, \cdots, 1)$ reveals only the cardinalities of the sets $S_{i} \cap S_{j}$.

The basic matrix equation (1.3) allows us to apply the powerful methods of matrix theory to the study of intersection patterns. It is of course difficult to make general statements concerning (1.3) without further constraints on the subsets. However, it is elementary to verify that

$$
\operatorname{rank}(Y)=\operatorname{rank}(A) \text {. }
$$

Indeed, an application of the standard theorems on rank to the matrix equation (1.3) implies

$$
\begin{aligned}
\operatorname{rank}\left(A A^{T}\right) & =\operatorname{rank}(Y(1,1, \cdots, 1)) \\
& \leqq \operatorname{rank}\left(Y\left(x_{1}, x_{2}, \cdots, x_{n}\right)\right) \leqq \operatorname{rank}(A) .
\end{aligned}
$$

But an arbitrary real matrix $Z$ has $\operatorname{rank}\left(Z Z^{T}\right)=\operatorname{rank}(Z)$, whence the result follows. We note further that in the special case $m=n$ we may take determinants in (1.3) and this gives

$$
\operatorname{det}(Y)=(\operatorname{det}(A))^{2} \prod_{i=1}^{n} x_{i}
$$

2. Applications. We now proceed with applications of the matrix equation (1.3) for subsets with various restricted intersection patterns.

THEOREM 2.1. Let $S_{1}, S_{2}, \cdots, S_{m}$ be subsets of the $n$-set

$$
S=\left\{x_{1}, x_{2}, \cdots, x_{n}\right\}
$$

and let $A$ of size $m$ by $n$ be the incidence matrix for these subsets of $S$. Suppose that the number of distinct nonempty set intersections of the form $S_{i} \cap S_{j}(i \neq j)$ is less than $n$. Then there exists an integral nonzero diagonal 
matrix $E$ and an integral diagonal matrix $D$ such that

$$
A E A^{T}=D
$$

Proof. We consider the matrix equation (1.3) for the subsets of Theorem 2.1. Let $t$ denote the number of distinct nonzero elements in $Y$ but not on the main diagonal of $Y$. These nonzero elements equated to zero give us a system of $t$ linear homogeneous equations in the $n$ unknowns $x_{1}, x_{2}, \cdots, x_{n}$. The matrix of this system of equations is a $(0,1)$-matrix in its own right. The assumption $t<n$ implies that the linear homogeneous system has an integral solution $e_{1}, e_{2}, \cdots, e_{n}$ with at least one $e_{i} \neq 0$. This integral solution yields the desired diagonal matrix $E$.

We digress briefly and describe some material from a forthcoming paper by Ryser [6]. Let $W$ denote a nonzero symmetric matrix of order $m \geqq 2$ with nonnegative integral elements and with 0 's in all of the main diagonal positions. Let $A$ be a $(0,1)$-matrix of size $m$ by $n$ and let each of the column sums of $A$ be greater than 1. Then we say that $A$ represents $W$ provided that there exists a diagonal matrix $D$ of order $m$ such that

$$
A A^{T}=D+W
$$

Thus $A$ may be regarded as the incidence matrix for subsets $S_{1}, S_{2}, \cdots, S_{m}$ of an $n$-set $S$ with the intersection cardinalities $\left|S_{i} \cap S_{j}\right|(i \neq j)$ prescribed by $W$. Extraneous elements are not allowed in the subsets in the sense that each of the elements of $S$ is required to occur in at least two of the subsets. It is entirely elementary to verify that there always exists a simple "canonical matrix" with column sums 2 that represents $W$. We designate by $C(W)$ the class of all $(0,1)$-matrices $A$ that represent $W$. Each of the matrices in $C(W)$ has exactly $m$ rows. But the number of columns may undergo considerable variation from one matrix to another.

A line of a matrix designates either a row or a column of the matrix. A triangle of a $(0,1)$-matrix $A$ is a submatrix of $A$ of order 3 with all line sums equal to 2 . The following simple lemma on triangles is derived in [5]. Let $A$ be a $(0,1)$-matrix of size $m$ by $n$. Suppose that every element of $A A^{T}$ is positive and that $A$ contains no triangles. Then $A$ contains a column of $m$ 1's. This lemma implies the following theorem concerning an arbitrary class $C(W)$ [6]. Every matrix in the class $C(W)$ contains a triangle or else the class contains exactly one matrix apart from column permutations without triangles.

We return to the incidence matrix $A$ of Theorem 2.1. Suppose now that each of the column sums of $A$ is greater than 1 . Then the matrix of our system of linear homogeneous equations does not contain a column of 0 's. Hence it follows that in this case the diagonal matrix $E$ contains both 
positive and negative $e_{i}$ 's. We associate the integer $e_{i}$ with column $i$ of $A$ and call $\left|e_{i}\right|$ the multiplicity of column $i$ of $A$. We now rewrite the matrix equation $(2.1)$ in the following form

$$
A_{1} A_{1}^{T}-A_{2} A_{2}^{T}=D \text {. }
$$

The matrix $A_{1}$ consists of the columns of $A$ with associated $e_{i}$ positive and each column is repeated with its appropriate multiplicity. Similarly, the matrix $A_{2}$ consists of the columns of $A$ with associated $e_{j}$ negative and each column is repeated with its appropriate multiplicity. The ordering of the columns within $A_{1}$ and $A_{2}$ is immaterial. Thus (2.3) tells us that $A_{1}$ and $A_{2}$ are both members of a certain class $C(W)$.

Now let us suppose further that the matrix $A$ is without triangles. Then both of the matrices $A_{1}$ and $A_{2}$ are also without triangles. Hence since $A_{1}$ and $A_{2}$ belong to the same class $C(W)$ we may conclude that $A_{1}$ and $A_{2}$ are the same apart from column permutations. Thus the matrix $A$ has two identical columns.

The following theorem is a direct consequence of the preceding discussion.

THEOREM 2.2. Let $A$ be a $(0,1)$-matrix of size $m$ by $n$. Let the columns of $A$ be distinct and let each of the column sums of $A$ be $\mathcal{E}^{r e a t e r}$ than 1. Suppose further that $A$ contains no triangles. Then $n \leqq m(m-1) / 2$.

Proof. Let us assume that $n>m(m-1) / 2$. Then the hypotheses of Theorem 2.1 are satisfied and equations (2.1) and (2.3) are valid. Furthermore, the assumption that $A$ contains no triangles implies that $A$ has two identical columns, contrary to hypothesis.

It is a remarkable fact that equality is attained in Theorem 2.2 for general $m$. We illustrate this for the case $m=5$. The following matrix

$$
\left[\begin{array}{llllllllll}
1 & 1 & 1 & 1 & * & * & * & * & * & * \\
1 & 0 & 0 & 0 & 1 & 1 & 1 & * & * & * \\
0 & 1 & 0 & 0 & 1 & 0 & 0 & 1 & 1 & * \\
0 & 0 & 1 & 0 & 0 & 1 & 0 & 1 & 0 & 1 \\
0 & 0 & 0 & 1 & 0 & 0 & 1 & 0 & 1 & 1
\end{array}\right]
$$

is of size 5 by 10 . Let the asterisks denote 0 's. Then the columns of the matrix may be regarded as all of the 2-subsets of a 5-set arranged in a natural ordering. This matrix has many triangles. But it is easy to verify inductively that every triangle must contain an asterisk. We now replace all of the asterisks by 1's. The new matrix is then without triangles and fulfills all of our additional requirements as well. 
The proof of the following theorem uses techniques similar to those employed by van Lint and Ryser [4] in their study of block designs with repeated blocks.

Theorem 2.3. Let $A$ be a $(0,1)$-matrix of order $n$ and let $A$ satisfy the matrix equation

$$
A E A^{T}=D .
$$

The matrices $D$ and $E$ are real (or complex) diagonal matrices of order $n$ and $D$ is nonsingular. Then $A$ is a permutation matrix.

Proof. Since the matrix $D$ is nonsingular we may write

$$
A E A^{T} D^{-1}=E A^{T} D^{-1} A=I,
$$

where $I$ is the identity matrix of order $n$. Hence it follows that

$$
A^{T} D^{-1} A=E^{-1} \text {. }
$$

An inspection of the main diagonal of (2.6) implies

$$
A^{T}\left(1 / d_{1}, 1 / d_{2}, \cdots, 1 / d_{n}\right)^{T}=\left(1 / e_{1}, 1 / e_{2}, \cdots, 1 / e_{n}\right)^{T} .
$$

We remark that in (2.7) we have made strong use of the fact that $A$ is a $(0,1)$-matrix. We now multiply (2.7) by $A E$ and this gives

$$
A(1,1, \cdots, 1)^{T}=(1,1, \cdots, 1)^{T} .
$$

Thus each of the row sums of $A$ equals 1 . But $A$ is a nonsingular $(0,1)$ matrix and hence $A$ is a permutation matrix.

\section{REFERENCES}

1. A. W. Goodman, Set equations, Amer. Math. Monthly 72 (1965), 607-613. MR 31 \#3337.

2. M. Hall, Jr., A problem in partitions, Bull. Amer. Math. Soc. 47 (1941), 804-807. MR 3, 166.

3. J. B. Kelly, Products of zero-one matrices, Canad. J. Math. 20 (1968), 298-329. MR 37 \#92.

4. J. H. van Lint and H. J. Ryser, Block designs with repeated blocks (to appear).

5. H. J. Ryser, Combinatorial configurations, SIAM J. Appl. Math. 17 (1969), 593-602. MR 41 \#1559.

6. - Intersection properties of finite sets (to appear).

Department of Mathematics, California Institute of Technology, Pasadena, California 91109 